\title{
Large-scale docking approaches to the kinome
}

\author{
Denis Schmidt*, Peter Kolb \\ From 9th German Conference on Chemoinformatics \\ Fulda, Germany. 10-12 November 2013
}

Docking nowadays is a widely used tool and has successfully and repeatedly been applied to identify hits with new chemical scaffolds [1]. Despite its successful applications, docking still suffers mainly form its scoring functions, which tend to be optimized for speed to the disadvantage of accuracy. Because of that, docking successfully enriches active molecules compared to inactive ones, however the false positive rate in docking runs is still very high. Several attempts have been made to improve docking by applying consensus scoring approaches using multiple structures or softwares or by rescoring compounds using normalization procedures [2-4]. At the same time, the number of large datasets of activity data derived from high-throughput screening methods has strongly increased during the last years, especially for kinases, allowing re-evaluation of these techniques [5-7]. We herein carried out a large-scale docking approach using 650 different kinase crystal structures. To the best of our knowledge, this is the largest number of targets docked to at the same time. Subsequently, different normalization techniques were re-evaluated using the experimental activity data as target function. Furthermore, we investigated the similarity of the kinase structures within the "docking universe" and correlated these similarities with other similarity metrices.

\section{Published: 11 March 2014}

\section{References}

1. Kolb P, Irwin JJ: Docking screens: right for the right reasons? Curr Top Med Chem 2009, 9:755-770

2. Vigers GPA, Rizzi JP: Multiple Active Site Corrections for Docking and Virtual Screening. J Med Chem 2004, 47:80-89.

3. Jacobsson M, Karlén A: Ligand Bias of Scoring Functions in StructureBased Virtual Screening. J Chem Inf Model 2006, 46:1334-1343.

4. Casey FP, Pihan E, Shields DC: Discovery of Small Molecule Inhibitors of Protein-Protein Interactions Using Combined Ligand and Target Score Normalization. J Chem Inf Model 2009, 49:2708-2717.

5. Karaman MW, et al: A quantitative analysis of kinase inhibitor selectivity. Nat Biotech 2008, 26:127-132.

* Correspondence: denis.schmidt@uni.marburg.de

Pharmaceutical Chemistry, Philipps-University, Marburg, Germany

6. Anastassiadis T, Deacon SW, Devarajan K, Ma H, Peterson JR: Comprehensive assay of kinase catalytic activity reveals features of kinase inhibitor selectivity. Nat Biotech 2011, 29:1039-1045.

7. Davis $\mathrm{Ml}$, et al: Comprehensive analysis of kinase inhibitor selectivity. Nat Biotech 2011, 29:1046-1051.

doi:10.1186/1758-2946-6-S1-P31

Cite this article as: Schmidt and Kolb: Large-scale docking approaches to the kinome. Journal of Cheminformatics 2014 6(Suppl 1):P31. 